\title{
Case study \\ The Nijmegen Method of Case Deliberation and Clinical Decision in a Multicultural Society
}

\section{Fayemi Ademola Kazeem PhD}

Lecturer, Department of Philosophy, Lagos State University, Nigeria \& Fellow, Scientific Institute for Quality of Healthcare, Radboud University, Nijmegen.

Email: kcaristotle@yahoo.com

\begin{abstract}
The Nijmegen method of ethical case deliberation is one of the ways of reflecting, clarifying, evaluating and making decisions about moral problems, conflicts and dilemmas in the clinical settings. It is a hybrid concentration of relevant ideas from different normative ethical traditions applied to the clinical practices. As a team multidisciplinary based deliberation, the method involves a professional ethicist serving as both a critical tutor and a facilitator in the process of deliberating and arriving at most ethical decision in clinical dilemmas. In such process, the Nijmegen method is not a democratic replacement of the decision and responsibility of the healthcare team. Rather it helps to motivate rational decision and reasoned responsibility in healthcare through consensus building which does not attenuate moral uprightness. The Nijmegen method is part of the healthcare process and aims at improving communication among patient, family and the healthcare team as well as enriching the decision making process. It is a promising method in resolving moral dilemmas in healthcare especially in multicultural societies. Besides enhancing the quality and transparency of the decision making process, ethical case deliberation on the ward using the Nijmegen method has prospect of serving as a baseline in guiding through similar dilemmas in the future.
\end{abstract}

Key words: case deliberation, Nijmegen method, clinical decision, multicultural society

Introduction: One of the key elements of the Nijmegen method of case deliberations is that it is a structured process involving consideration of all the basic factors, dimensions and inventory of facts relevant to a clinical case. As a multidisciplinary deliberative process, the method is differentiated into four steps ${ }^{1}$ : (i) the moral problem, which consists of actual moral problem implicit in the case; (ii) the inventory of the facts in the case: diagnosis, therapy, and prognosis of the patient; nursing care, selfcare, patient's activities of daily living, patient's values (social, religious, cultural and psychosocial dimensions), the institutional and legal dimensions relevant to the case; (iii) weighing of moral values and norms, which include the patient's well-being, respect for autonomy, informed consent, respect for life, representation by proxy, question of justice, responsibility of the health care professionals and the team; and (iv) decision making with justifiable reasons and putting such decision into action. 
In this piece, an attempt is made in providing a post-reflection on a clinical case using the Nijmegen method of ethical case deliberation on the ward. The aim is to: (i) explore how the case would have been deliberated upon peradventure there was clinical ethics committee in the hospital where the case happened; (ii) proffer prospective baseline in guiding through similar dilemmas in future clinical decisions anywhere it may occur.

Ethical Considerations: Informed consent procedure was followed in the collection and reportage of the data. In line with the terms of consent agreement reached with the family, the identities and locations of family members, the traditional physician, and the clinical laboratory were veiled in order to ensure confidentiality. Only the researcher listened to the audio recording.

Case: Mrs. X, a Yoruba woman in her early forties who was fourteen weeks pregnant, developed a severe headache on her return from the market. Her husband, Mr. X, massaged her head with the traditional herbal balm, which did not relieve her. The intensity of her condition till the following day made $\mathrm{Mr} . \mathrm{X}$ to call on the herbalist who promised to make some spiritual healing ritual the subsequent day if the herbal medication he brought did not relieve Mrs. $X$ from the headache. Some hours later that night Mrs. $X$ suddenly lost vision in one eye; on the intervention of their neighbours, she was taken to the hospital in the city rather than the herbalist.

A CT scan of Mrs. X's head revealed an intracerebral hemorrhage with massive swelling which put pressure on the brainstem. The option of surgical operation has $50 \%$ chance of survival, so revealed the physician. Upon hearing this, Mr. X reached out to family members who consented to the surgery and rallied round for financial support. The operation was done without complications. But the neurosurgeons found no explanation for the etiology of her hemorrhage; neither was there evidence of an aneurysm, a tumor, a vascular malformation, nor hypertension or clotting disorder. The neurosurgeons sent necrotic brain tissue to the pathology for analysis.

In the ICU Mrs. $X$ did not regain consciousness as she was put under mechanical ventilation. On the next day, a repeat CT scan showed a stroke in the opposite hemisphere from the hemorrhage. In explaining these new findings to $\mathrm{Mr}$. $\mathrm{X}$ and some family members in presence through the help of an interpreter, the doctor reported her poor prognosis. She became hypertensive that night and required medication to maintain her blood pressure. While not contesting any of the clinical actions, the family members pulled further resources to allow the 'city technologists' save Mrs. X. In the two-day hospitalization of Mrs. X, there was a steady-stream of about 20-30 family members, and friends in the visitors' lounge wanting to see her. This situation did not go down well with the nurses who repeatedly disclose the ICU policy of no more than two visitors at the bedside at a time.

On the third day, an obstetrician did a fetal ultrasound that showed evidence of a pregnancy but no fetus. This led him to the conclusion that either Mrs. X had had a miscarriage or was less than seven weeks pregnant. Mr. $\mathrm{X}$ was mystified by the ultrasound result as he openly disclosed that his wife had 
been pregnant for more than three months and there was no sign of miscarriage. There, in the presence of the family, nurses and the physicians, Mr. $\mathrm{X}$ disclosed that the pregnancy was as a consequence of a traditional herbal contraceptive failure as they never wanted to have another child in addition to their sixth children. However, they have both contemplated on abortion, which they never did after all. On this confession, some family members speculated that the couple's thoughts about abortion of the fetus might have spiritually caused the illness of Mrs. X. The family therefore agreed to perform some traditional rituals and oral medicinal treatments that could keep her soul, dissolve clot and release brain swelling. To burn incense as part of the ritual, they needed the doctor's permission to turn off the supplementary oxygen in her room in the ICU.

The doctor did not agree to the request of performing ritual in the ICU let alone discontinuing the oxygen. Upon return to the hospital the following day to further persuade the doctor, the doctor through the assistance of a trained interpreter told the family that Mrs. X was brain-dead. The nurses made request about organ donation, which one of the family members said this could not be tabled before the family because of their cultural beliefs about it. Mr. X complained that had the physician agreed with the family's request on complementary traditional healing, his wife would not have died with her pregnancy. While leaving in grief, the family members asked the doctor to remove the fetus because it is not culturally allowed to bury two persons in the same grave. When they were shown the ultrasound picture of an empty uterus again, the family members were confused; however, many of them recourse to spiritual etiology in understanding the absence of the fetus: the fetus's soul had caused the deceased illness and then had left her without any vaginal bleeding.

However, the pathologist's later report indicated the presence of placental tissue in Mrs. X's brain. The physician's explanations for her hemorrhage was that she had a cancer of the placenta that had spread from the uterus and undeveloped fetal tissue to the brain, causing the intracerebral hemorrhage.

\section{The Nijmegen method of ethical analysis of case deliberation}

Following the Nijmegen protocol of case deliberation in the clinical setting, the case of Mrs. $X$ is analyzed as follows:

\section{a. What is the moral problem?}

Does the doctor have a moral duty to concede to the family's request of turning off the patient's mechanical ventilator in order to allow traditional healing ritual in the ICU?

\section{b. Inventory and interpretation of facts:}

1. Medical dimension (diagnosis, therapy and prognosis): The patient was pregnant, lost vision in one eye and had severe headache, which the husband thought initially that massaging with herbal balm in-line with traditional self-medication practices would cure. But first clinical diagnosis showed 
that the patient's severe headache was as a result of bleeding in the brain. Emergency intracranial surgery was performed and the patient later became hypertensive with a stroke in the opposite hemisphere of the hemorrhage. She developed apnea and was sustained by mechanical ventilator. Though with a pregnancy of more than three months, the ultrasound conducted and interpreted by the obstetrician showed pregnancy without fetus. While there was delayed understanding of the etiology of the patient's condition, which caused serious crisis of confidence in the whole clinical care process among the patient's family despite their believe in the potency of 'the city technology', the later report of the pathologist cleared the air as it showed that the patient had placental cancer. The patient's prognosis was poor as she was in coma being sustained with ventilator in the ICU. Given the late pathological diagnosis of Mrs. X condition, her chance of neurological recovery was slim as her comorbidity assessment showed that not only did she have undeveloped fetal tissue because of the cancer of the placental, her medical condition is also not within curative limits as the cancer had spread from the uterus to the brain, causing severe intracerebral hemorrhage, which eventually resulted in her brain-death. Her brain cells and reflexes are dead.

2. Nursing dimension: The nursing team did not have a smooth and companion relationship with the patient's family as they challenged many of the Yoruba traditional practices throughout Mrs. X's brief and painful stay in the hospital: long waiting at the emergency department; sticking strictly to the hospital policy of restricted number of visitors at the bedside at a time; dismissing the family's explanations of other possible etiology of the patient's condition; inviting organ donation even under the emotional tormenting and grief moment without anticipation of cultural and personal outrage. The family's experience of the nursing team was one of hopelessness and professional routine controlled. The nursing team prevented the overcrowding of the patient's bed in order to ensure control, avoid distraction and allow bed-rest, which they thought was in the best interest and care of the patient.

3. Patient's view (values, religious, psychological and cultural dimensions): The patient was an illiterate and not immersed in orthodox medicine. Giving the rural setting of the patient's environment, she had more access to traditional herbal medical care than orthodox medicine in clinical setting. This is evident in the kind of contraceptive patronized and the consequent failure in preventing unwanted pregnancy after six children. She had no clinical diagnosis of her pregnancy nor was there prenatal treatment in a hospital setting. The patient had preference for traditional medicine and there are strong indications that she believed in the efficacy of religious rituals. However, the possible lack of state-ofthe-art diagnosis and medical check-ups in the traditional medical practices, which she is culturally accustomed to may have accounted for why the cancer of the placenta was not early diagnosed.

4. Social dimension: The social context of the case is very important because the medical dilemma and tensions happened in a multicultural context of different understanding of care, death, organ donation practices, privacy, and informed consent, which altogether played out, negatively, in the patient, family, healthcare team relationships. The patient and her family are from a culture that honors communal solidarity together with the complementariness of the ancient medicinal wisdom with 
western orthodox medicine. This accounted for why the family agreed taking Mrs. $\mathrm{X}$ to the hospital with the hope that the 'city medical technology' would save her. But the healthcare professions did not share that sense of mutual complementarily as they conceived clinical practices as a necessary antithesis of traditional medical practices. This conflict played out in the case.

The financial aspect also comes in as evident in the family social cooperation in pooling resources in order to pay the bills of their wife, sister, cousin and friend. In addition, the patient's family belief about the spiritual repercussion of mere contemplation of abortion even without actually carrying it out showed the strong value the Yoruba attach to sanctity of human life from conception. While such taboo was discountenanced by the scientifically-minded physician, the patient's family believed it was the cause of her condition. Though the physician with the assistance of the professional translator in the hospital tried to convince Mrs. X family that she was dead, the family had some difficulties initially in believing that she was dead not until the mechanical ventilator was removed.

5. Organizational dimension: The clinical setting is a private hospital in a multicultural society with diverse languages and ethnic groups. The hospital has team of professional translators who facilitate communication between the healthcare team in the hospital and patients, who cannot communicate in the official English language. This notwithstanding, there is little evidence that the hospital management recognized that non-Western-values immersed patients often experience the orthodox healthcare system as invasive, frightening and antithetical to their aged-long practice of traditional indigenous medical care. For instance in the case, the hospital policy did not reckon with the solidarity culture of many well-wishers being around a patient.

6. The legal aspect: Currently, while there are laws regulating each healthcare system separately, there is no legislation in Nigeria on integrative healthcare such that compels the clinical physician to allow the application of rituals, herbs and other healing processes by the traditional herbal practitioners in a clinical setting. Clinical guidelines in government owned hospitals prohibit ritual performance in the hospital; however, it is accepted in few private hospitals depending on the perception of the owner. Therefore, in this case, the doctor's decision and refusal of the request to turn off the oxygen on the patient in order to allow traditional healing ritual in the ICU is not illegal.

\section{c. Moral values and norms:}

1. Well-being of patient: While the healthcare team wanted to promote the well-being of the patient by means of the best healthcare, which the physician interpreted as keeping the patient on ventilator, the family also wanted to promote the patient's well-being by requesting to be allowed to complement the Western clinical healthcare with the traditional healing process. But there was a conflict between the physician and the family in this process of promoting the patient's well-being. On the one hand, the physician's actions and decisions were based on the duties of beneficence (e.g. the prompt the surgical intervention) and maleficence (e.g. protection of the patient from harm that may result by 
keeping her off-oxygen). On the other hand, the family's decision was taken on the basis of care hinged on solidarity.

There were differences in the family's and physician's perceptions of the etiology of Mrs. X's condition and that influenced different ideas about the needed care for her well-being. These differences in perspectives caused misunderstanding and mistrust which at the end, did not promote the patient's well-being. Giving the awareness that there was little left to offer Mrs. X, a perspective may suggest that the physician should have agreed to the family request of complementary traditional healing in the ICU. Another view may in fact counter-argue that the patient's relatives ought not in the first instance to have taken the patient to the hospital with its own different clinical guidelines which are not fully in tandem with the family's understanding of etiology, diagnosis and prognosis.

After being declared brain-dead, the family believed that even at death, the patient's well-being must be taken into consideration by respecting the dignity of her body. The expectation in this regard is that keepng her on the ventilator is an abuse of the body; burying her with the fetus, which they believed was still in the patient constitute a taboo; and organ donation of any of her bodily part was understood not only a terrible humiliation but also a bodily loss.

2. Autonomy of the patient and informed consent: Up to the pre-surgery moment, Mrs. $X$ was conscious but was not as concerned about her autonomy as getting relieved from her severe headache and sight loss condition. Given the communal nature of the Yoruba, proxy and extended consent play greater role than patient's autonomy and informed consent. The husband together with the family consensually took decision in the patient's best interest.

3. Responsibility within the team of health care professionals: There seem to be a good team working relationship among the health care professionals: doctor, nurses, obstetrician, pathologist, and the interpreter. However, none of them responded well to pertinent aspects of patient's cultural practices, values and beliefs in traditional medicine in the clinical setting. The traditional medicinal practitioner was seen as a non-collaborative agent that they owe no responsibility in the caring process of the patient.

d. Conclusion and decision making on the case: In view of the facts of this case, it is evident that "cultural differences may create barriers to the delivery of care." When such differences are not prudently handled, they result into moral dilemmas and distress not only for the healthcare team, but also for the patient and the family caregivers. It is therefore important that "healthcare providers identify and address possible cultural barriers at the levels of organizational management and clinical practices." ${ }^{2}$ In multicultural and ethnic societies, healthcare providers need to be open to combined therapeutic approaches to patient well-being. The Western engrossed healthcare team in the case of Mrs. X was intolerant of possible complementary indigenous medical practices, which Mrs. X's family believed could have saved her life. The imprecision and late clinical understanding of the actual 
etiology of Mrs. X's condition underestimated the much trust and confidence the patient and her family had in Western clinical practices.

While it is arguable that the doctor has a moral duty to promote the patient's best interest in the face of poor prognosis by allowing the traditional healing process to be conducted alongside the clinical treatment (even when there is no guarantee of the outcome of the traditional healing intervention), such duty was beclouded by the absence of dialogue and lack of respect for alternative medicinal model. Though there was communication as transmitted by the translator, there was no substantive dialogue and trusting relationship between the family caregivers, patient and the healthcare team. A genuine dialogue between the doctor and the family may have given rise to rational compromise, which may have saved the patient's brain death. The paternalistic posture of the doctor was not beneficent for the well-being of the patient. Fundamental to good patient care and family's satisfaction with the health services is sensitivity to patient's healthcare beliefs, practices and shared preferences with the physician.

\section{References}

1. Steinkamp N, Gordijn B. Ethical case deliberation on the ward: a comparison of four methods. Med Health Care Philosophy, 2003; 6(3): 235-46.

2 Culhane-Pera, K. et. al. (eds.) Healing by Heart. Nashville: Vanderbilt University Press, 2003.

Conflict of Interest: The author has no conflict of interest.

Acknowledgments: My profound gratitude goes to Professor Norbert Steinkamp and Mrs. Anifowose Seun for their valuable suggestions. I thank the Erasmus Mundus Master of Bioethics Programme for the opportunity of my participation in the 2013/2014 edition of the programme 